\title{
Autonomous Assistance Control based on Inattention of the Driver when Driving a Truck Tract
}

\author{
Elvis Bunces and Danilo Zambrano \\ Universidad de las Fuerzas Armadas ESPE, Sangolquí, Ecuador \\ \{eabunces, vdzambrano\} despe. edu.ec
}

\begin{abstract}
This article proposes the autonomous assistance of a Truck based on a user's inattention analysis. The level of user inattention is associated with a standalone controller of driving of assistance and path correction. The assistance algorithm is based on the kinematic model of the Truck and the level of user inattention In addition, a 3D simulator is developed in a virtual environment that allows to emulate the behavior of the vehicle and user in different weather conditions and paths. The experimental results using the virtual simulator, show the correct performance of the algorithm of assistance proposed.
\end{abstract}

Keywords: Driver's Inattention, Truck, Drivers of assistance, Car-like

\section{Introduction}

The transit accidents attribute grave problems in today's society interfering directly with the global economy. This particularly affects countries low economic income, where $65 \%$ of injuries in the population are attributed to these accidents [1,2], registering that the last two decades the current social transformation, migration and industrialization, they locate the developing countries as the most in having high rates of car accident and it is estimated that each year die 1.25 million people around world [3]. However, the mortality rate among users of rural roads highly developed countries is lower in comparison to the countries en paths developing [4] this is how transit accidents are a priority problem in public health for the World Health Organization (WHO) in terms of high mortality rates and economic costs that have been generated in recent years [5]. Previous studies have identified some main factors that are directly related to this phenomenon, as are: negligence, noncompliance with traffic laws and lack of attention when driving, showing that the latter is the leading cause of accidents of transit on road $[6,7]$.

The driving of vehicles entails to perform specific tasks and in some cases with a degree of complexity at different levels and unlimited time scales the attention, coordination and concentration play an important role in skills of driving to prevent road accidents. However, the driver must not only do this work but also carry out secondary tasks how to observe the GPS, talk on a cell phone, etc. Regardless of any activity that might attract your attention, this causes stress in the driver, that can disturb your attention, and generate affectations not only personal but in other cases to third per- 
sons [8]. For this reason, different accident prevention techniques have been put into practice with the aim of tackling the problematic presented, among them are have artificial or intelligent vision systems, these techniques are denominates ITS (Intelligent Transportation Systems), the new generation of safety systems is made up mainly of innovative technologies commensurate to the performance of the vehicles of in the actuality [9]. These systems detect driver fatigue and can easily react in real time with an audible signal. [10], which not only fit into two seater or family vehicles but are also implemented in public transport vehicles, mining and heavy transport, obtaining from the latter the longest time a driver is behind the wheel at extended times with an estimated up to 15 hours of continuous driving according to the NHTSA (National Highway Traffic Safety Administration) resulting in a high level of fatigue and drowsiness, $[11,12]$

The ITS systems and auditory alerts, if well propose a preventive in car accidents, they have been generated drawbacks with the type of alert they issue, the loud noise and location of the emitter, are some causes that have caused an instinctive reaction of the motor reflex at the moment of driving that are reflected in potential accidents $[13,14]$. One of the tools currently available to deal with these problems is the use of driving simulators, which are indistinctly designed with scenarios to evaluate a driver's characteristics in the face of unexpected events that also occur in real driving. On the other hand, researchers have shown that drivers modify their behavior according to the risk they perceive, thus defining an analysis of a driver's inattention and reaction to a visual alert. [15].

Taking into account the analysis and problems presented above, this document is defined with the purpose of analyzing the inattention of a user by means of the realization of a driving simulator of a vehicle type Truck in virtual reality. Coupling to this an autonomous path correction control; the simulator is designed in such a way that it generates an immersion to the user identical to the driving on the road and feedback of vibratory and axial forces emitted by the haptic device. In addition, the haptic control devices of the vehicle, they fulfill the same function as the devices controls of a real vehicle, and allows an evaluation of the user's performance when performing driving maneuvers.

\section{Virtual Environment with Driving Simulator}

Fig. 1 shows how the software and hardware components are linked. The inattention control program, uses information about the movement of the user's head, provided by the virtual device Óculus Rift.

The stage of simulation of the scene in Unity 3D contains all the programming of virtual reality, where the 3D model of the Truck and the haptic input devices are linked with the physical and kinematic properties of vehicle movement; The stage of 
SCRIPTS, manages communication with haptic devices and 3D Model of the vehicle, providing the virtual environment with the required functionality.

QuNITY 3D

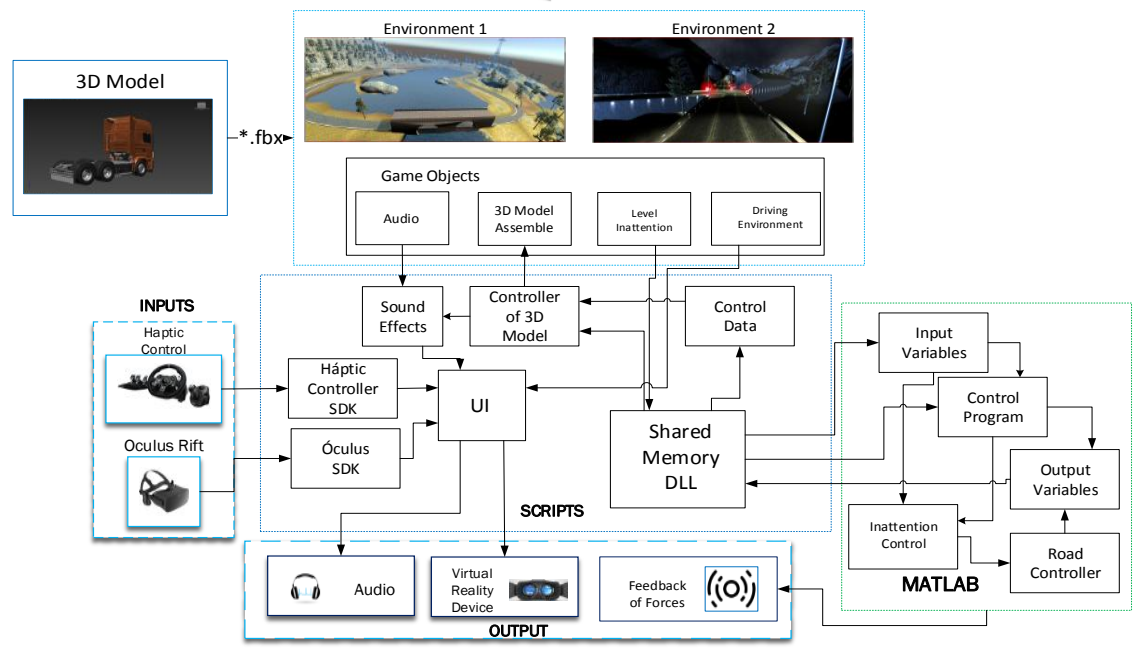

Fig 1. Operative Scheme

In addition, position, velocity and orientation variables are shared bidirectional with Matlab through a shared memory. The output phase provides the user surround audio, a virtual environment sensitive to the movement of scenes, haptic response with feedback of vibratory forces and axial forces generated by the path control. The design of the 3D model of the Truck starts in a CAD software, which is a tool that generates on detail solids 3D. In Fig. 2, the multilayer scheme for the development of applications in virtual environments is shown with the aim of providing greater immersion to users in driving tasks.

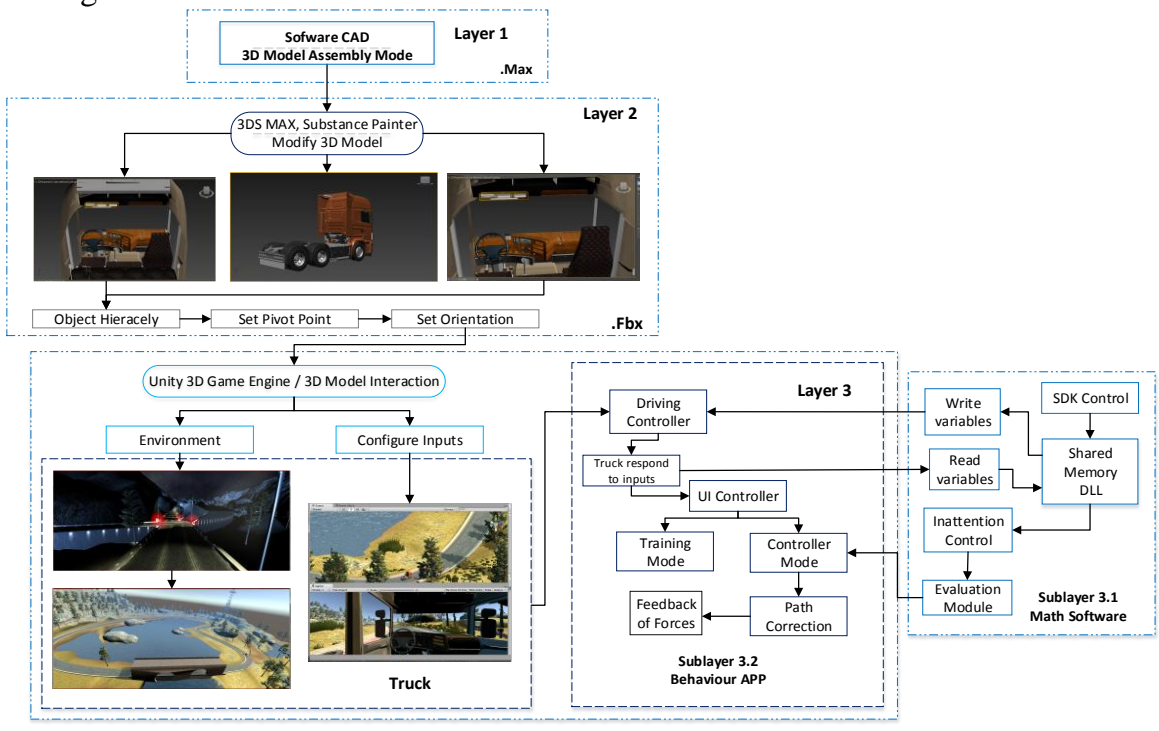

Fig 2. Multi-layer diagram 

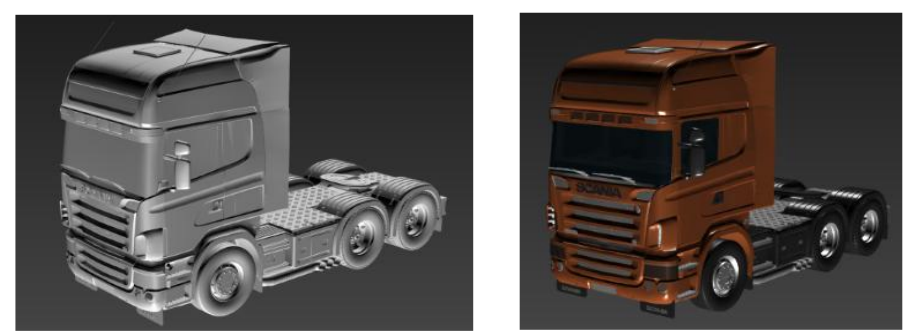

Fig 3. Truck assembled

Layer 2: in this layer the control elements (steering wheel, pedal board, gear lever and propulsion axes) are determined from the reference system, which is arranged in a hierarchical manner, allowing characteristic movements. In addition, materials and textures are applied to each component of the vehicle to increase its detail to the user; Layer 3: In this layer, the 3D model with the kinematics and texture defined, is imported into the Unity $3 \mathrm{D}$ scene, each component is linked to the control and data entry algorithms. In Fig.4. The vehicle is shown in two virtual scenarios created to perform driving maneuvers.
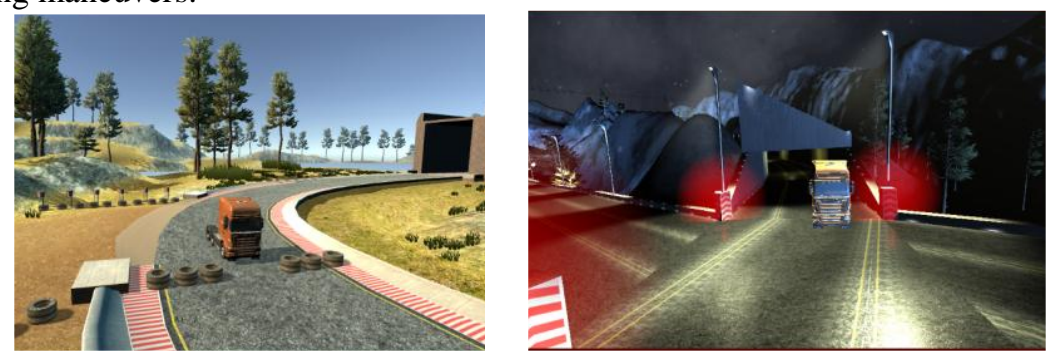

Fig 4. Driving scenes

In addition, there are control sub-layers that are described as follows; Sub-layer 3.1: Matlab mathematical software contains the mathematical modeling and control law that analyzes the level of user attention and manages the control of driving assistance; Sub-layer 3.2: This sub-layer has the logical programming of control and analysis of data that is obtained with respect to the driving of the user, resulting in auditory responses, visual alerts, and feedback of axial and vibratory forces from the steering wheel to the user.

\section{Controller Design}

The control of paths tracking, although it is a subject very used in the case of the robots type car-like, in this section, an autonomous assistance algorithm is proposed based on: visual inattention of the user; mathematical model of the Truck; and in the paths tracking problem.

\section{a) Kinematic Modeling}

The kinematic model of the Truck, consider the point of interest $h_{T}=\left(x_{T}, y_{T}\right)$ at a distance $a$ of the rear axle wheels propulsion, to the center of the vehicle, as shown in Fig. 5. 


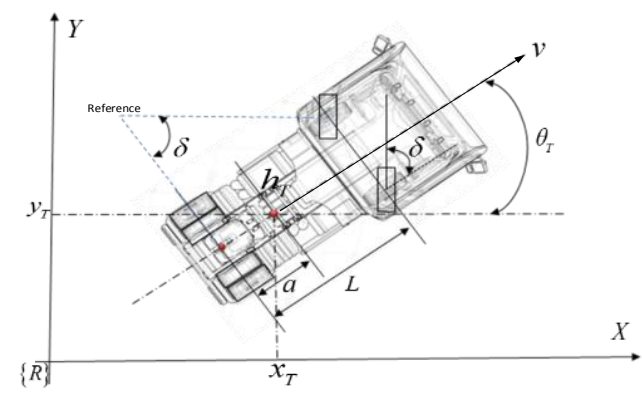

Fig 5. Kinematic model of the car-like truck

From Fig.5 the kinematic model of the Truck is defined

$$
\left\{\begin{array}{l}
\dot{x}_{T}=\mu \cos \left(\theta_{T}\right)-a \omega \sin \left(\theta_{T}\right) \\
\dot{y}_{T}=\mu \sin \left(\theta_{T}\right)+a \omega \cos \left(\theta_{T}\right) \\
\dot{\theta}_{T}=\omega=\frac{v}{L} \tan (\delta)
\end{array}\right.
$$

The cinematic model (1), It can be expressed in a compact form as:

$$
\begin{aligned}
\dot{\mathbf{h}}_{T}(t) & =\mathbf{J}\left(\theta_{T}\right) \mathbf{v}(t) \\
\dot{\theta}_{T}(t) & =\frac{\mu}{L}\left(\theta_{T}\right) \mathbf{v}(t)
\end{aligned}
$$

where $\mathbf{J}\left(\theta_{T}\right)$, is the Jacobiana matrix, that defines a rectilinear mapping between the velocity vector $\mathbf{v}(t)$ of the Truck, and $\dot{\mathbf{h}}_{T}(t)$ it is the final vector of the control speeds, with respect to the reference system $\{R\}$

b) Path Specification

In Fig. 6, the path correction problem is shown, represented by $P(s)$ where, $P(s)=\left(x_{p}(s), y_{p}(s)\right) ; h_{d}(s)=\left(x_{p}\left(s_{d}\right), y_{p}\left(s_{d}\right)\right)$ is the current desired point of the Truck, which is considered as the closest distance to $P(s)$; the profile of errors, in the orientation $X$, is given by $\tilde{x}=x_{p}\left(s_{d}\right)-x$; and in the orientation $Y$ is given by $\tilde{y}=y_{p}\left(s_{d}\right)-y$

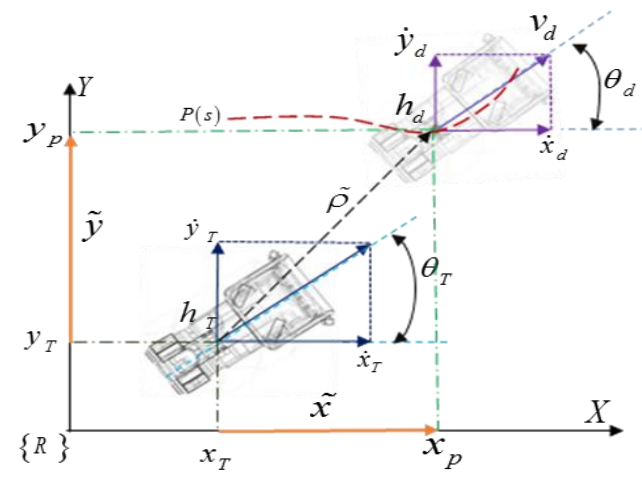

Fig 6. Path correction model

Based on the graph of the Fig.6, the control errors $\rho(t)$, are deducted by the difference in position, between the current point of the Truck $h(x, y)$ and the desired point $h_{d}$, where the distance between the current position of the vehicle $h(x, y)$ and the 
reference point, it's zero $\tilde{\rho}=0-\rho=-\rho ; \tilde{\theta}=\theta_{p}\left(s_{d}\right)-\theta_{T}$, where $\theta_{p}\left(s_{d}\right)$ is the orientation of the unitary vector that is tangent to the path $h_{d}$ in relation to the reference system $\{R\}$

c) Definition of the Desired Velocity

For consideration of Truck velocity, the manipulation of the desired speed is proposed, depending on different quantifications, i.e., driving errors; curvature of the path; inattention index.

$$
v_{d}(t)=\frac{v_{\max }}{1+k_{1}\|\tilde{\rho}\|+k_{2}\|\Upsilon\|}
$$

where, $v_{\max }$ is the maximum speed desired on the chosen path; $k_{1} ; k_{2}$ represent constants that ponder the error and the radius of curvature $\Upsilon$ of the desired path. When considering a path $P$ as an aggregate of points, the curvature value is defined as:

$$
\Upsilon(k)=\frac{|\dot{\mathbf{P}}(k) \times \ddot{\mathbf{P}}(k)|}{|\dot{\mathbf{P}}(k)|^{3}}
$$

The values of the radius curvature in each time interval of (4), can only be found if you have the analytical expression of the path. This limits to a large extent the use of this type of considerations, since for real applications the route to follow is not always available in the form of derivable mathematical equations. To solve the limitation of not having the analytical expression, it is proposed to use the following point $\mathbf{P}(k+1)$ and the previous point $\mathbf{P}(k-1)$ of the sampling cycle, in this way, $\dot{\mathbf{P}}(k)$ is determined in this case as: $\dot{\mathbf{P}}(k)=\frac{\mathbf{P}(k-1)-\mathbf{P}(k+1)}{2 T_{s}}$ and the $\ddot{\mathbf{P}}(k)$ value is calculated by:

$$
\ddot{\mathbf{P}}(k)=\frac{\mathbf{P}(k+1)-2 \mathbf{P}(k)+\mathbf{P}(k-1)}{T_{s}^{2}}
$$

d) Definition of the Inattention Index

The inattention index is based on the driver's vision area, with respect to the visible area of the path and the angle of movement of the head inside the cabin of the Truck.

$$
i_{p}(t)=1-\frac{A_{\text {path }_{i}}(t)}{A_{\text {path }_{\max }}(t)}
$$

where : $A_{\text {path }_{i}}(t)$; is the user's vision intersection area, within the visible section of the path in the direction of vehicle movement and it depends on the driver's angle of vision, shown in Fig. 7; $A_{\text {path }_{\max }}(t)$; is the maximum area of intersection that exists between the visible area of the driver and the path in real time i.e.

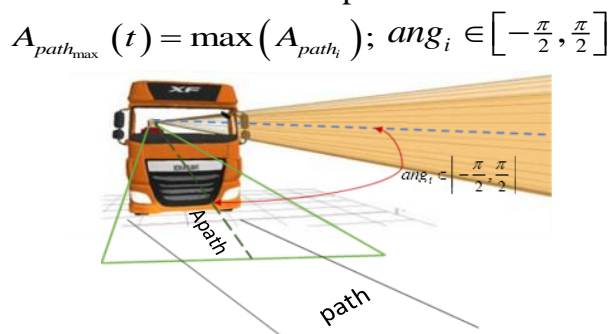

Fig 7. Area of vision 


\section{e) Assistance Control Design}

The proposed kinematic controller design, they are based on numerical methods tools. Particularly for the solution of systems of equations, these systems can be constituted in matrix form, for which theorems and axioms of linear algebra are applied.

Considering the first order differential equation

$$
\dot{\mathbf{h}}(t)=f(\mathbf{h}, \mathbf{v}, \mathbf{\Gamma}) \text { with } \mathbf{h}(\mathrm{O})=\mathbf{h}_{\mathrm{o}}
$$

where, $\mathbf{h}$ represents the output of the controller system; $\dot{\mathbf{h}}$ it is the first derivative with respect to time; $\mathbf{v}$ is the control action; and $\mathbf{\Gamma}$ represents different driving criteria. The values of $h(t)$ in time discrete $t=k T_{0}$ they are called $h(k)$, where $T_{0}$ represents the sampling time and $k \in\{1,2,3,4,5 \ldots\}$ in addition, the use of numerical methods for calculating system progression, is based on the possibility of bringing the system closer to a state of time $k$, if the state, and the control action are known at the moment of time $k-1$, this approach is called Euler's method. Hence, the design of the kinematic controller is based on the kinematic model of vehicle. In order to design the kinematic controller, the model of the Truck (2) can be approximated as:

$$
\frac{\mathbf{h}_{T}(k)-\mathbf{h}_{T}(k-1)}{T_{0}}=\mathbf{J}\left(\theta_{T}(k)\right) \mathbf{v}(k)
$$

Taking into account that the path correction which consists of locating the vehicle within a predefined path without parameterization in time. Therefore the control objective is to position the desired point, at the closest point of the path $P(s)$ at a desired velocity $v_{d}$. To have a scope of the exposed objective the following expression is considered:

$$
\frac{\mathbf{h}_{T}(k)-\mathbf{h}_{T}(k-1)}{T_{\mathrm{o}}}=v_{d}(k)+\mathbf{W}\left(\frac{\mathbf{h}_{\mathbf{d}}(k-1)-\mathbf{h}_{T}(k-1)}{T_{\mathrm{o}}}\right)
$$

where, $\mathbf{h}_{\mathbf{d}}$ is the desired path, $\mathbf{W}\left(\tilde{\mathbf{h}}_{T}(k-1)\right)$ is a diagonal matrix that control error weights, defined as: $\mathbf{W}\left(\tilde{h}_{T m}(k-1)\right)=\frac{w_{m}}{1+\left|\tilde{h}_{T m}(k-1)\right|}$ where $m$ represents the operational coordinates of the vehicle. Now, to generate the system equations consider (7) and (8), the system can be rewritten as $\mathbf{A u}=\mathbf{b}$

$$
\underbrace{\mathbf{J}\left(\theta_{T}(k)\right)}_{\mathbf{A}} \mathbf{v}(k)=\underbrace{i_{p}(k) \mathbf{v}_{\mathbf{d}}(k)+\frac{\mathbf{W}\left(\mathbf{h}_{\mathbf{d}}(k-1)-\mathbf{h}_{T}(k-1)\right)}{T_{0}}}_{\mathbf{u}}
$$

Whereas the Jacobiana matrix $\mathbf{J} \in \boldsymbol{R}^{m \times n}$ has the same number of unknowns as equations $(m=n)$ with rank $r=n$ for each $\mathbf{b} \in R^{m}$, then (9) represents a linear system with general solution.

$$
\mathbf{v}_{\text {ref }}=\underbrace{\mathbf{J}\left(\theta_{T}(k)\right)^{-1}\left(i_{p}(k) \mathbf{v}_{\mathbf{d}}(k)+\frac{\mathbf{W}\left(\mathbf{h}_{\mathbf{d}}(k-1)-\mathbf{h}_{T}(k-1)\right)}{T_{0}}\right)}_{\mathbf{v}_{1}}+\underbrace{g_{i}(k) \mathbf{v}_{\mathbf{h}}(k)}_{\mathbf{v}_{2}}
$$

where, $g_{i} \in[0,1]$ is defined as $g_{i}(k)=1-i_{p}(k)$; and $\mathbf{v}_{\mathbf{h}}(k)=\left[\begin{array}{lll}u_{h}(k) & \omega_{h}(k)\end{array}\right]^{T}$ is the vehicle's maneuverability vector, generated by man. The proposed control law consists of two main terms $\mathbf{v}_{\text {ref }}=\mathbf{v}_{1}+\mathbf{v}_{2}$, where, $\mathbf{v}_{2}$ represents the driver's maneu- 
verability to the vehicle in relation to the inattention rate $i_{p}$, i.e., The greater the inattention of the driver, the lower the incidence of the maneuverability signals generated by the user through the haptic devices; while $\mathbf{v}_{1}$ is the term in charge of correcting the control errors produced when the vehicle does not follow the desired path i.e., when there is user inattention the desired velocity of movement of the vehicle is weighted in relation to $i_{p}$. In conclusion when the index of inattention $i_{p}$ increase $\mathbf{v}_{2}$ decreases and $\mathbf{v}_{1}$ it increases, which ensures that the vehicle does not get out of the path desired what could cause an accident.

f) Feedback of Forces

To generate the feedback of forces in the steering wheel the equation is used (11), that describes the relationship between lateral forces $F_{x, y}$, that are generated on the wheels of direction when taking a curve, in relation to the torque applied on the steering wheel to correct the orientation of the Truck.

$$
M_{v}=\frac{M_{r}}{r d(\eta)}
$$

Where $M_{v}$ is the moment on the steering wheel, $M_{r}$ defines the torque that exists in the wheels of steering, $r d$ the transmission ratio with regard to the steering angle of the steering wheel vs., the angle of rotation of the wheels and $\eta$, is the performance of the direction of the Truck. According to the equation (11) it defines: $M_{r}=F_{x, y}\left(r_{w}\right)$ with $F_{x, y}=\frac{m_{T}\left(\mathbf{v}_{r e f}\right)}{\Upsilon}$ whereby $r_{w}$, is the radius of the wheels; $m_{T}$ It is the mass of the Truck; $r$, represents the radius of curvature of the path and the equation (10), $\mathbf{v}_{r f}$ you get the current operating velocity of the Truck. i.e., $M_{v}$ it's the torque that is exercised in the steering wheel, according to each curve that present in the driving.

\section{g) Stability Analysis}

For the stability analysis, the most critical case is considered, i.e., when the user's inattention level is the maximum $i_{p}=1$, therefore (10) depends solely on $\mathbf{v}_{\mathbf{1}}$ It is also considered perfect velocity tracking $\mathbf{v}_{\text {ref }}(t)=\mathbf{v}(t)$, (7) so it can be replaced in the kinematic model (7) on (10), obtaining the following closed loop equation:

$$
\frac{\mathbf{h}_{T}(k)-\mathbf{h}_{T}(k-1)}{T_{\mathrm{o}}}=\underbrace{\hat{r}}_{i_{p}(k) \mathbf{v}_{\mathrm{d}}(k)+\frac{\mathbf{W}\left(\mathbf{h}_{\mathrm{d}}(k-1)-\mathbf{h}_{T}(k-1)\right)}{T_{\mathrm{O}}}}
$$

It is considered that $i_{p}(k) \mathbf{v}_{\mathbf{d}}(k)=\mathbf{v}_{\mathbf{d}}(k)+\dot{\boldsymbol{\eta}}(k)$, In addition, the signal is defined $\dot{\gamma}$ as the difference between $\dot{\mathbf{h}}_{\mathrm{d}}$ and $\mathbf{v}_{\mathrm{d}}$, i.e.

$$
\begin{gathered}
\mathbf{v}_{\mathbf{d}}(k)=\dot{\mathbf{h}}_{\mathbf{d}}(k)+\dot{\gamma}(k) \\
\mathbf{h}_{T}(k)-\mathbf{h}_{T}(k-1)=T_{0}\left(\left(\dot{\mathbf{h}}_{\mathbf{d}}(k)-\dot{\gamma}(k)+\dot{\boldsymbol{\eta}}(k)\right)+\frac{\mathbf{W}\left(\mathbf{h}_{\mathbf{d}}(k-1)-\mathbf{h}_{T}(k-1)\right)}{T_{0}}\right) \\
\mathbf{h}_{T}(k)-\mathbf{h}_{T}(k-1)=T_{\mathrm{o}}\left(\frac{\mathbf{h}_{\mathbf{d}}(k)-\mathbf{h}_{\mathbf{d}}(k-1)}{T_{0}}+\frac{\Delta \boldsymbol{\eta}}{T_{0}}+\frac{\Delta \gamma}{T_{0}}+\frac{\mathbf{W}\left(\mathbf{h}_{\mathbf{d}}(k-1)-\mathbf{h}_{T}(k-1)\right)}{T_{0}}\right)
\end{gathered}
$$

The control error is defined as: $e(k-1)=\mathbf{h}_{\mathbf{d}}(k-1)-\mathbf{h}(k-1)$, thus 


$$
e(k-1)=e(k)+\mathbf{W}(e(k-1))-\Delta \gamma-\Delta \boldsymbol{\eta}
$$

if $\Delta \xi=\Delta \gamma+\Delta \boldsymbol{\eta}$, so:

$$
\begin{gathered}
\Delta \xi=e(k)-e(k-1)+\mathbf{W}(e(k-1)) \\
\Delta \xi=e(k)+e(k-1)(\mathbf{W}-1)
\end{gathered}
$$

For this case, the transformed of $\mathrm{z}$ applies:

$$
\begin{gathered}
\left(1-z^{-1}\right) \xi(z)=e(z)+e(z) z^{-1}(\mathbf{W}-1) \\
\left(1-z^{-1}\right) \xi(z)=e(z)\left(1+z^{-1}(\mathbf{W}-1)\right) \\
e(z)=\frac{1-z^{-1}}{1+z^{-1}(\mathbf{W}-1)} \xi(z)
\end{gathered}
$$

the poles of the system (13) are;

$$
1+z^{-1}(\mathbf{W}-1)=0
$$

So that the poles of the system (13) are within the unit radius then, it is necessary that the profit matrix $0<\mathbf{W}\left(\tilde{h}_{T}(k-1)\right)<1$ Thus, in this way it is concluded that control errors $\tilde{\mathbf{h}}(k)=0$ when $k \rightarrow \infty$, has asymptotic stability, that is to say, the vehicle follows the desired path when there is a level of inattention of the user when driving.

\section{Experimental Results}

In this stage, conduction tests for the purpose of evaluate the performance of the control of assistance based on the user's inattention while driving Truck in virtual reality. In Fig. 8 the experiments performed are shown, for this, an HP laptop is used (AMD Dual-Core, 3GB RAM, 500GB HDD), Óculus Rift, Headphones, Logitech steering wheel (G920 Force Feedback Racing), pedals and gear lever
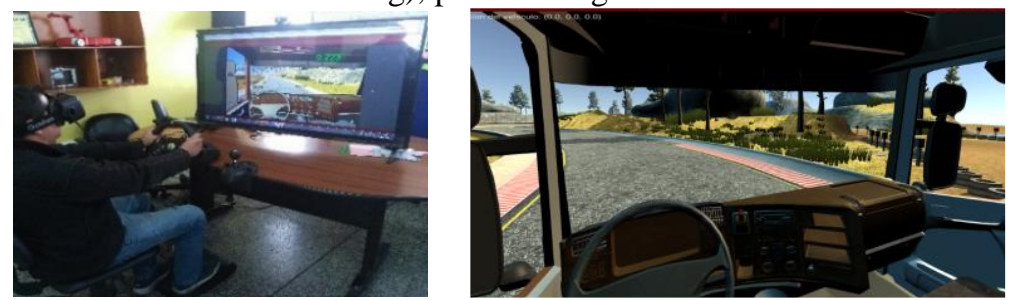

Fig 8. Driving test

In Fig. 9, a menu is displayed in which the user can select two types of driving stage, the first stage is generated with a night driving environment, vs., to the second scenario that has a driving in the day.

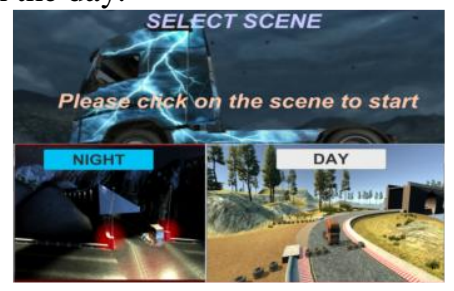

Fig 9. Select scene 
Fig. 10 shows the relationship between the desired path $v s$, the path executed by the user, where (a) shows the path executed with the autonomous driving assistant deactivated and in (b) the driving assistant is activated and permanently monitors every action of the driver for correction the orientation and velocity of the Truck.

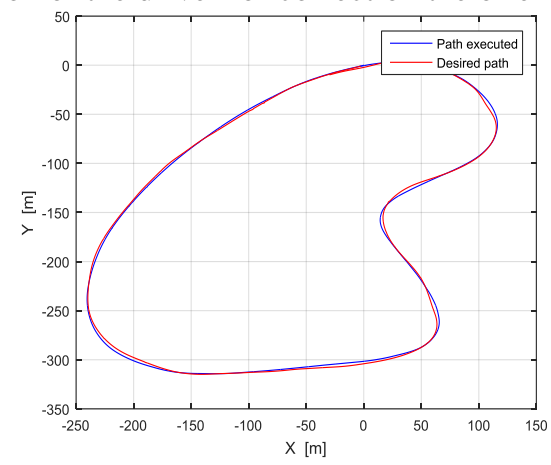

(a) Without driving assistance

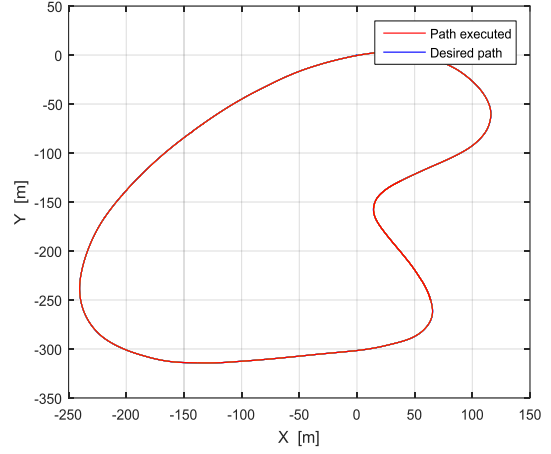

(b) With controller of driving

Fig 10. Path executed

The inattention index presented by the driver during the driving test phases is measured permanently in a continuous numerical range: $(0,0.1,0.2,0.3 \ldots \ldots .1)$, where, 0 , represents an efficient level of care, until 1, which represents a higher level of inattention. As shown in Fig. 11.

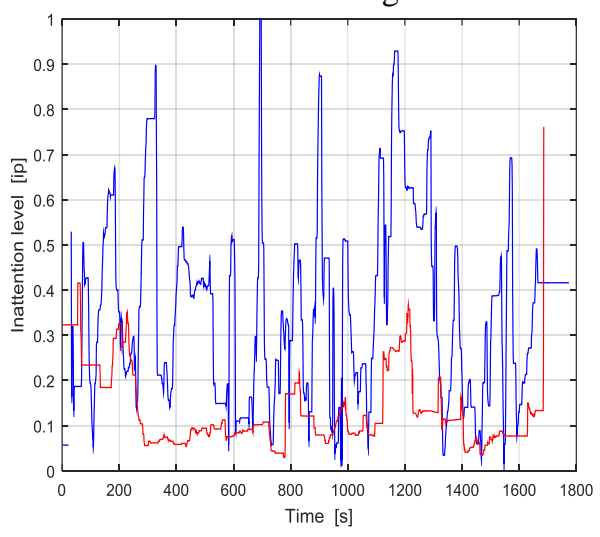

Fig 11. Inattention level

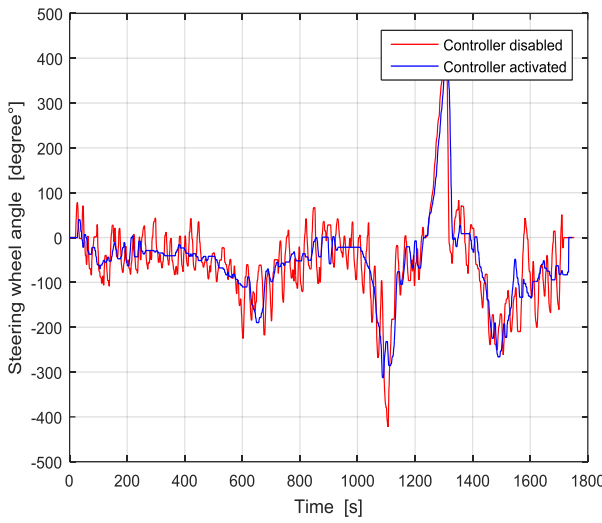

Fig 12. Steering wheel angle

To perform the ideal path correction, from the driver's steering wheel, by feedback of axial forces, a ratio adjustment is used, between the steering angle of the steering wheel and the angle of rotation of the wheels, i.e., for a Truck the total turn angle of the direction $\gamma_{w}$, is $900^{\circ}$, and the blocking angle $\lambda_{b}$, of the wheels is $30^{\circ}$, where the following formula is applied $\lambda_{b} \frac{\gamma_{w}}{2}$ obtaining as a result a ratio of $r d=15: 1$, , that for every $15^{\circ}$ of turn of steering wheel, the wheels will turn $1^{\circ}$. The trajectory control results are shown in Fig. 12, in which a conduction is carried out with absence of the driving controller and another with the driving assistant activated, and it is observed that the axial force feedback, controls the rotation of the steering wheel continuously along the desired trajectory. 
The velocity printed by the driving assistant to the Truck can be seen in Fig. 13, where it maintains a controlled speed during the journey, and when the driver exceeds the velocity limit, the controller reduces the velocity autonomously. And you get as a result, an efficient control of the velocity compared to a driving without a controller.

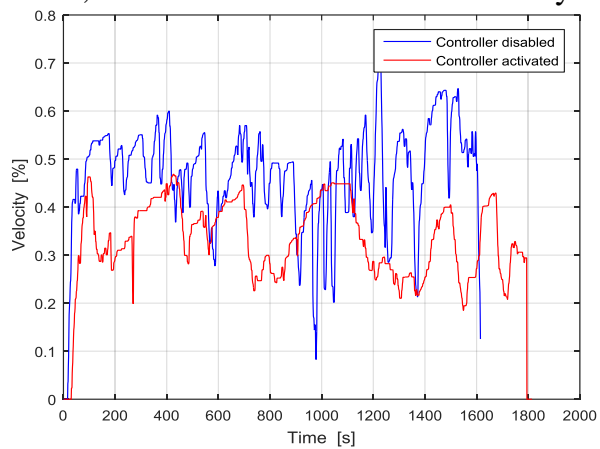

Fig 13. Controller of velocity

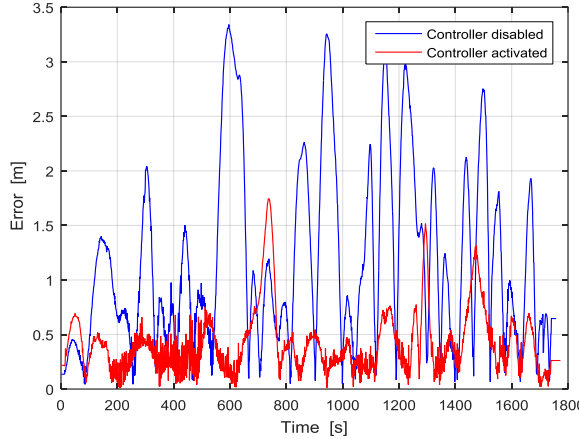

Fig 14. Path error

Fig. 14, shows the error that was obtained with the driving assistant activated, during the performance of driving maneuvers with a Truck in virtual reality, $v s$. a driving with the driving assistant turned off, therefore the loss of trajectory was greater.

When the user turns his head out of the area of the visible section of the path, the inattention detection system, sends a visual warning signal to the driver as shown in Fig. 15.

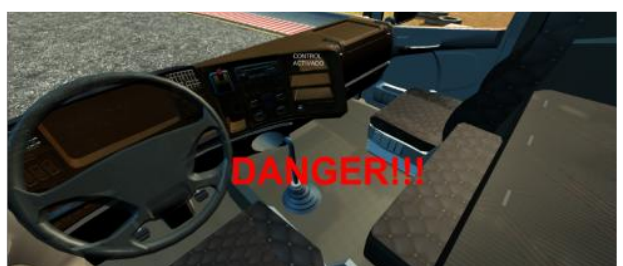

Fig 15. Inattention warning sign

\section{Conclusions}

This document presented a design that analyzes the inattention index that a driver has when performing driving maneuvers, and the design of a driving assistant, based on control of tracing of paths car-like, that helps the user to obtain better driving habits. The high degree of detail added in the 3D model, increases driving immersion to the user. The advantage of this, is the ability to perform driving maneuvers similar to driving on the road, with the uniqueness of being a Truck. So, focusing on the application, from the point of view of driver training, in this type of vehicle of heavy transport, it becomes a tool for students who are learning to drive a vehicle following the conventional educational process. The use of virtual reality has benefits from a secure environment, where the driving tests are also monitored by the driving assistant, which records the ability to maneuver this vehicle, even in situations of risk, allowing to know the level of concentration that the driver can have, when facing this type of situations, that are part of a daily driving on the road. 


\section{References}

1. P. Shruthi, V.T. Venkatesh, B. Viswakanth, C. Ramesh, P.L. Sujatha, I.R. Domonic. "Analysis of Fatal Road Traffic Accidents in a Metropolitan City of South India", In J Indian Acad Forensic Med, vol. 35, no. 4, pp. 317-320, (2013).

2. Farooqui J.M., Chavan K.D., Bangal R.S., Syed M.M.A., Thacker P.J., Alam S., Sahu S., Farooqui A.A.J., Kalakoti P. "Pattern of injury in fatal road traffic accidents in a rural area of western Maharashtra”, In India, Australasian Medical Journal, pp. 476-482, (2013).

3. Ali Davoudi-Kiakalayeh, Reza Mohammadi, Shahrokh Yousefzade-Chabok, Sohiel Saadat. "Road traffic crashes in rural setting: an experience of a middle-income country", In Chinese Journal of Traumatology, vol. 17, pp. 327-330, (2014).

4. Moafian G., Aghabeigi M.R., Hoseinzadeh A., Lankarani K.B., Sarikhani Y. "An epidemiologic survey of road traffic accidents in Iran: analysis of driver- related factors", In Chinese Journal of Traumatology, vol. 16, pp. 140-144,(2013).

5. Montes S.A., Introzzi I.M., Ledesma R.D., López S.S. "Selective Attention and Error Proneness whilw Driving: Research Using a Conjunctive Visual Search Task", Avances en Psicología Latinoamericana, vol. 34, pp. 195-203, (2016).

6. Danilo Chavez G., Emanuel Slawinski., Vivente Mut. "Modeling the Inattention of a Human Driving a Car", 11th IFAC/IFIP/IFORS/IEA Symposium on Analysis, Design, and Evaluation of Human-Machine Systems, vol. 43, pp. 7-12, (2010).

7. Terry C. Lansdown., Amanda N. Stephens., Guy H. Walker. "Multiple driver distractions: A systemic transport problem", In Accident Analysis \& Prevention Magazine, vol. 74, pp. 360-367, (2015).

8. Kristie L. Young., Paul M. Salmon. "Sharing the responsibility for driver distraction across road transport systems: A systems approach to the management of distracted driving", InAccident Analysis \& Prevention Magazine, vol. 74, pp. 350-359, (2015)

9. Klaus Bengler, Klaus Dietmayer, Berthold Färber, Markus Maurer, Christoph Stiller, Hermann Winner. "Three Decades of Driver Assistance Systems", In IEEE Intelligent Transportation Systems Magazine, pp. 6-22, (2014).

10. Stephen M. Casner., Edwin L. Hutchins., Don Norman. "The challenges of partially automated driving", In Magazine Communications of the ACM N, vol. 59, pp. 70-77, (2016).

11. Jeff K. Caird., Katherine A. Jhonston., et aT. "The use of meta-analysis or research synthesis to combine driving simulation or naturalistic study results on driver distraction", In Journal of Safety Research Magazine, vol. 49, pp. 91-96, (2014).

12. Despina Stavrinos., Jennifer L Jones., et aT., "Impact of distracted driving on safety and traffic flow", In Accident Analysis \& Prevention Magazine, vol. 61, pp. 63-70, (2013).

13. Tiffany L. Overton., Terry E. Rives., et aT. "Distracted driving: prevalence, problems, and prevention", In International Journal of Injury Control and Safety Promotion, vol. 22, pp. 187-192, (2015).

14. Lleneras Robert E., Salinger Jeremy., Green Charles A. "Human Factors Issues Associated with Limited Ability Autonomous Driving Systems: Drivers' Allocation of Visual Attention to the Forward Roadway”, In Proceedings of the Seventh International Driving Symposium on Human Factors in Driver Assessment, Training and Vehicle Desing, pp. 92-98, (2013).

15. Myungwook Park., Sangwoo Lee., Wooyong Han. "Development of Steering Control System for Autonomous Vehicle Using Geometry-Based Path Tracking Algorithm" In ETRI Journal, vol. 37, no. 3, pp. 617-625, (2015). 